\title{
UESCOPE AS A METHOD OF ENDOTRACHEAL INTUBATION OF TRAUMA PATIENT
}

\author{
Wladyslaw Gawel ${ }^{1}$, Halla Kaminska ${ }^{2}$, Wojciech Wieczorek ${ }^{3}$ \\ ${ }^{1}$ Student Scientific Association of Children's Diabetology at Medical University of Silesia, Katowice, Poland \\ ${ }^{2}$ Department of Children's Diabetology, Medical University of Silesia, Katowice, Poland \\ ${ }^{3}$ Department of Anaesthesiology, Intensive Care and Emergency Medicine in Zabrze, Medical University of Silesia, Katowice, Poland
}

Disaster Emerg Med J 2017; 2(4): 175-176

\section{Dear Editor,}

Endotracheal intubation is one of the basic forms of protecting airway patency during both cardiopulmonary resuscitation and in trauma patients $[1,2]$. A severe head injury often disturbs one's consciousness and impairs one's ability to maintain airway patency. Immobilization of the cervical spine that does not require the use of external devices includes manual stabilization of the cervical spine and head. However, the most preferred method during emergency is immobilization with a cervical collar. Numerous studies indicate that endotracheal intubation in such conditions may be difficult [3-5], due to the inability to bend the head backwards and to position a patient with a cervical spine injury into a sniffing position. Another limitation is the difficult opening of the oral cavity when using standard cervical collars. In the case of direct laryngoscopy, i.e. using a laryngoscope with a Miller or Macintosh blades, intubation in the cervical collar decreases the effectiveness of the first intubation attempts, which then results in the need to perform subsequent attempts prolonging the time when the patient is not being ventilated. Continuous attempts of endotracheal intubations may lead to the development of 'vicious circle' phenomenon when each unsuccessful endotracheal intubation may result in bleeding and airway oedema inducing the phenomenon described by the Difficult Airway Society as "can't intubate, can't ventilate" [6]. In this situation, the only option to maintain airway patency is through needle cricothyroidotomy. Hence, in order to avoid such situation, it is crucial to search for alternatives of direct laryngeal endotracheal intubation which will increase the effectiveness of the first intubation attempt in trauma patients.
The study was designed as a prospective, randomized cross-over study and was carried out from November to December 2017. The study involved 63 final year medicine students participating in emergency medicine classes. Participation in the study was voluntary. Before their inclusion in the study, all participants had completed their anaesthesiology course and had declared the ability to perform endotracheal intubation based on direct laryngoscopy. The study was preceded by training of maintaining airway patency with a Macintosh laryngoscope and a UEScope video laryngoscope (UEScope; UE Medical Devices, Newton, MA, US). After completing the theoretical part, the instructors demonstrated the correct intubation procedure using the above-mentioned devices, following which all participants of the study had the opportunity to perform 20 endotracheal intubations utilizing the MAC and UEScope on normal airways. The real study took place one week after theoretical and practical training. During the evaluation, study participants were asked to perform endotracheal intubation using the MAC and UEScope in the conditions of immobilized cervical spine (Fig. 1). Both the order of intubation methods and participants were randomized. To simulate a traumatic patient, a Resusci Anne training manequin (Laerdal Stavanger, Norway) was used.

The success rate of the first endotracheal intubation attempt with MAC usage was $23.8 \%$ and was statistically significantly lower than in the case of intubation with the UEScope $(53.9 \%, p<0.001)$. The average intubation time while utilizing the analyzed devices varied between $42 \pm 17 \mathrm{~s}$ for the MAC and $34 \pm 11 \mathrm{~s}(\mathrm{p}=0.023)$ for the UEScope. Consequently, $95.2 \%$ of all the study participants declared that 

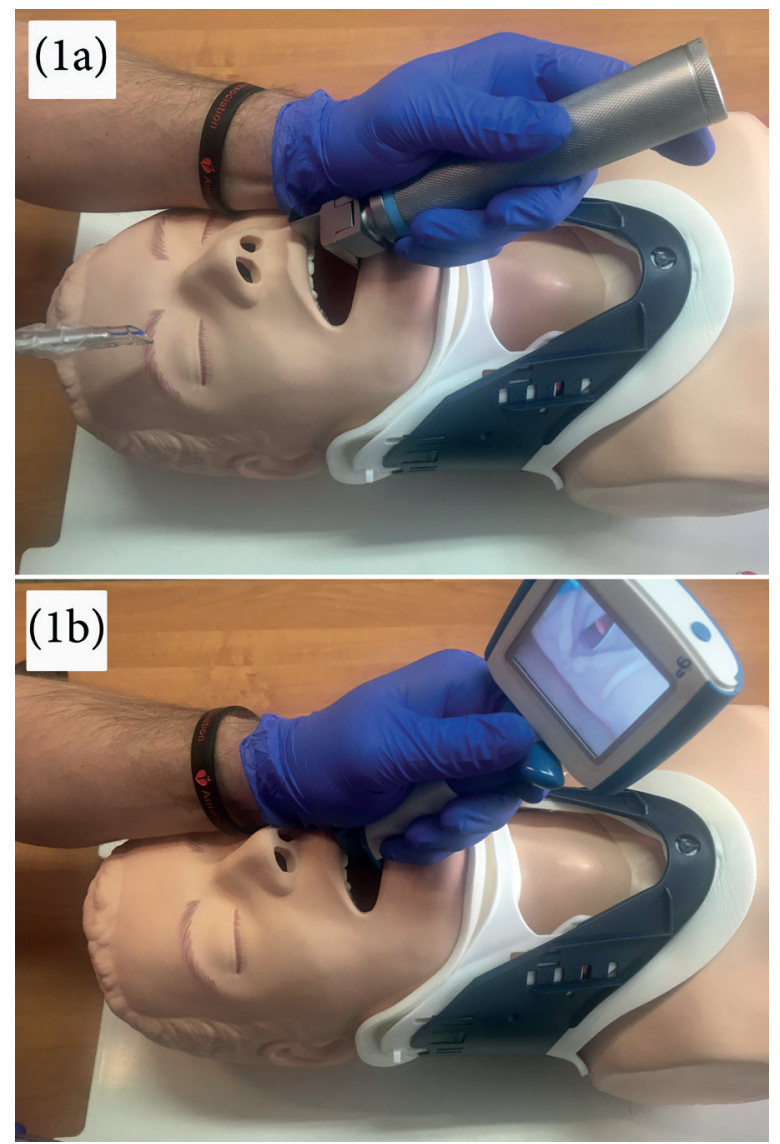

FIGURE 1. (1a) Intubation using Macintosh laryngoscope; (1b) Intubation using UEScope video laryngoscope

they would prefer to perform intubation using the UEScope laryngoscope in real-life conditions.

To summarize, the present study shows that final year medical students performed endotracheal intubation with the usage of UEScope with a higher efficacy and in a shorter time in patients with cervical spine immobilization. However, further studies are required with other medical professionals to confirm these results.

\section{REFERENCES}

1. Kim DH, Yoo JY, Ha SY, et al. Comparison of the paediatric blade of the Pentax-AWS and Ovassapian airway in fibreoptic tracheal intubation in patients with limited mouth opening and cervical spine immobilization by a semi-rigid neck collar: a randomized controlled trial. $\mathrm{Br}$ J Anaesth. 2017; 119(5): 993-999, doi: 10.1093/bja/aex272, indexed in Pubmed: 28981579.

2. Karczewska K, Szarpak L, Smereka J, et al. ET-View compared to direct laryngoscopy in patients with immobilized cervical spine by unexperienced physicians: A randomized crossover manikin trial. Anaesthesiol Intensive Ther. 2017; 49(4): 274-282, doi: 10.5603/AlT.a2017.0047, indexed in Pubmed: 28953308.

3. Wang SY, Xue FS, Yang GZ, et al. Performance of C-MAC videolaryngoscope for intubation in cervical spine immobilization conditions. Am J Emerg Med. 2017 [Epub ahead of print], doi: 10.1016/j. ajem.2017.10.052, indexed in Pubmed: 29074069.

4. Klosiewicz T, Sip M, Zalewski R, et al. Alternative of endotracheal intubation for paramedics to provide direct laryngoscopy. The randomized manikin trial. Disaster Emerg Med J. 2017; 2(3): 142-144, doi: 10.5603/DEMJ.a2017.0032.

5. Frass M, Robak O, Truszewski Z, et al. Comparison of endotracheal intubation with the Airtraq Avant ${ }^{\circledR}$ and the Macintosh laryngoscope during intermittent or continuous chest compression: a randomized, crossover study in manikins. Disaster Emerg Med J. 2016; 1(1): 7-13, doi: 10.5603/DEMJ.2016.0002.

6. Gawlowski P, Smereka J, Madziala M, et al. Comparison of the ETView Single Lumen and Macintosh laryngoscopes for endotracheal intubation in an airway manikin with immobilized cervical spine by novice paramedics: A randomized crossover manikin trial. Medicine (Baltimore). 2017; 96(16): e5873, doi: 10.1097/MD.00000000000005873, indexed in Pubmed: 28422820. 